\title{
REALIDAD ETNOEDUCATIVA EN UNA COMUNIDAD MAPUCHE: UN ANÁLISIS DESCRIPTIVO ${ }^{(1)}$
}

\author{
Alberto Moreno Doña \\ Universidad Santo Tomás, La Serena, Chile
}

\section{INTRODUCCIÓN}

En la investigación educacional adquiere mayor presencia la educación formal y la educación no formal, relegándose a un segundo plano la educación informal $\left({ }^{2}\right)$. Esta educación informal vendría a ser el proceso de socialización que los niños vivencian al exterior de la escuela o aquellos aprendizajes efectuados en la escuela a partir del llamado currículo oculto.

La mayoría de los teóricos educacionales se han dedicado a estudiar los aspectos que influyen en el aprendizaje originado al interior de las escuelas, olvidando o postergando el estudio de aquellos factores que causan el aprendizaje en los procesos informales, como por ejemplo el aprendizaje de la lengua materna.

Estos resultados hacen pensar y cuestionarse la separación existente entre el proceso de educación informal y el proceso escolar o proceso formal educativo. La transmisión de principios de enseñanza aprendizaje de la educación informal a la escuela podría generar ciertos cambios en las instituciones educativas formales, los cuales ayudarían a aminorar los malos resultados académicos que hasta ahora se están obteniendo (UNESCO, 2005) $\left(^{3}\right)$.

Es aquí donde empezamos a cuestionarnos una serie de premisas que nos parecen fundamentales: ¿Qué tiene el proceso de socialización que es capaz de generar mejores aprendizajes que el proceso escolar en sí? ¿Por qué los científicos y educadores no se han dedicado a indagar cómo surge el aprendizaje en ese proceso de socialización? ¿Qué le ocurre a la escuela que no es capaz de generar aprendizajes de calidad? ¿Cómo es posible que culturas con tanta complejidad y riqueza como las indígenas sean capaces de conseguir en sus neófitos un aprendizaje de calidad en relación con su cultura? ¿Qué de positivo posee ese aprendizaje informal que fuera susceptible de ser trasladado a la escuela institucionalizada?

La exclusión del estudio teórico, por parte de los investigadores educativos, de la educación informal pareciera estar originada en la dificultad de analizar sus aspectos caóticos, ambiguos, ambivalentes, paradojales e inciertos, que queramos o no, son fruto de aprendizajes exitosos en el medio informal. Al

\footnotetext{
${ }^{1}$ Este artículo forma parte del Proyecto de Investigación FONDECYT 1030147/2003: "La educación desde la teoría del caos: potencialidad de la educación y limitación de la escuela", financiado por el Fondo Nacional de Investigaciones Científicas y Tecnológicas del Consejo Nacional de Ciencia y Tecnología de Chile (CONICYT) y dirigido por el Dr. Carlos Calvo Muñoz.

2 "La educación ocurre a lo largo de la vida y no se limita a la escuela. Cuando ocurre en ambientes escolarizados se le llama 'educación formal' o 'educación no formal'. Cuando sucede fuera de la escuela se le denomina educación informal y corresponde a la socialización o enculturación. Estas distinciones son erróneas e inducen a serios errores sobre las características y potencialidades de la educación. La educación formal es cartesiana en su fundamento y orientación, al igual que la educación no formal, que trata de actividades de capacitación y animación socio cultural, breves, dinámicas, flexibles y certificables. Sus participantes, a diferencia de la escuela, tienen orígenes, edades y actividades diferentes que se reúnen por objetivos específicos y temporales, quienes se separan, una vez finalizada la capacitación. La educación informal representa los procesos educativos que tienen lugar en la calle, el café o el salón escolar" (Calvo, 2002)
} 
menos así lo muestran algunas investigaciones y propuestas recientes (Calvo, 2002).

La educación informal comparte los aspectos comentados con la etnoeducación, la cual no hay que confundir con la educación indígena institucionalizada. Esta última, tendría œmo uno de sus objetivos la integración asimilacionista del indígena a la 'cultura nacional', basándose prácticamente en la castellanización de tales indígenas. Se podría decir que la educación oficial indígena consiste en la educación de los indígenas sin su participación. Por el contrario, la etnoeducación es la educación propia de cada etnia, que si bien es diferente de unas a otras, comparten ciertos aspectos que suponemos son universales. Uno de los aspectos distintivos, entre otros $\left({ }^{4}\right)$, es la no linealidad del proceso de enseñanza aprendizaje.

Es desde esta premisa de donde partimos para pensar que un estudio descriptivo de la etnoeducación nos podría ayudar a develar algunas premisas fundamentales del proceso de enseñanza aprendizaje en ese proceso informal, 'natural', que ocurre al interior de las culturas indígenas de Chile. Este trabajo de investigación se centra en un contexto indígena -el mapuche- y no en nuestro contexto, debido al éxito que aquel ha tenido para enseñar sus aspectos propios sin la necesidad de una escuela institucionalizada.

La presente investigación tiene como objetivo:

Describir el proceso etnoeducativo en una comunidad mapuche

\section{MÉTODO}

\section{Diseño}

La presente investigación es un estudio cualitativo de carácter descriptivo. La metodología cualitativa se refiere a procedimientos que posibilitan una construcción de conocimiento sobre la base de conceptos. Desde aquí, la presente investigación en su carácter descriptivo utiliza una perspectiva inductiva, es decir, de lo particular a lo general. Los procedimientos de análisis empleados, en general se ciñeron a las propuestas de fragmentación y articulación de la "grounded theory" (Glaser \& Strauss, 1967; Srauss \& Corbin, 1990). Se realizó codificación abierta, con categorías emergentes; y codificación axial, en torno a la agrupación de las categorías emergentes en familias de categorías. La codificación selectiva, y por tanto el trabajo interpretativo, aunque se realizó, no será planteado en este artículo.

\section{Unidad de análisis}

La unidad de análisis de esta investigación corresponde a la comunidad rural mapuche del sector Collico Ranco en la Región de la Araucanía de Chile. Su ubicación exacta es Camino Carahue - Puerto Saavedra Km 14

El número de sujetos participantes en las diversas modalidades de recolección de datos fue diferente. Observaciones etnográficas se realizarón a los niños y niñas de la Escuela Municipal de Collico Ranco. Estas observaciones siempre se limitaron a contextos de aprendizaje informales. Entrevistas en profundidad se aplicaron a cuatro adultos mapuches (dos hombres y dos mujeres) y un niño mapuche, a

${ }^{3}$ UNESCO (2005). Education for All Global Monitoring Report 2005 - The Quality Imperative. Consultado el 4 de enero de 2005 en: http://portal.unesco.org/education/en/ev.php-URL ID=35939\&URL DO=DO TOPIC\&URL SECTION=201.html entre otras

${ }^{4}$ Características del proc eso de enseñanza - aprendizaje informal y etnoeducativo serían la ambigüedad, incertidumbre, 
una machi y a dos profesores no mapuches de escuelas de educación intercultural mapuches y se optó por utilizarla, por dos razones específicas: en primer lugar para que los resultados de las entrevistas fueran de la mano de los datos obtenidos en el método etnográfico, y en segundo lugar, para interpretar en forma correcta los acontecimientos observados y que generaron dudas al investigador a partir de dicho método (Taylor \& Bogdan, 1984).

El proceso seguido para afianzar la credibilidad, consistencia y confirmabilidad de la investigación es, en la investigación cualitativa, la triangulación. El tipo de triangulación utilizada en esta investigación ha sido el juicio de experto, para lo que hemos contado con la colaboración del Dr. Francisco Gutiérrez, de la Universidad de La Salle de Costa Rica, y la Dra. Virginia Ferrer, de la Universidad de Barcelona, España, además de la utilización de diferentes métodos en el mismo objeto de estudio.

\section{Fases del procedimiento de recolección y análisis de datos}

1. Diseño y planificación del estudio. Incluye contacto con los profesores, alumnos y apoderados de la Escuela Collico Ranco de la Región de la Araucanía, observaciones etnográficas para ponerme en contacto con el proceso a estudiar y su contexto, y elaboración de los guiones para las entrevistas en profundidad.

2. Implementación del estudio. Consistió en la realización de los registros etnográficos en su totalidad y en la aplicación de las entrevistas en profundidad.

Las observaciones etnográficas se realizaron durante los días 3 y 28 de agosto del 2004 en distintos horarios, los cuales fluctuaron desde las 8.30 hasta las 17.00 horas. Se eligieron los periodos de recreo y/o descanso en los que los alumnos/as ocupaban los diferentes espacios de recreación de la escuela. Los alumnos/as estaban en conocimiento de mi labor como investigador.

Las entrevistas en profundidad se realizaron durante el mes de agosto de 2004. Durante las entrevistas solo nos encontrábamos el investigador y el entrevistado. Las entrevistas tuvieron diferentes duraciones pero rondaron, como tiempo promedio, los 45 minutos. Antes de iniciarse cada una, a cada entrevistado se le solicitó permiso para grabarla, accediendo todos a tal petición.

3. Análisis e informe de los resultados. Se realizó el registro de la información, su codificación y análisis descriptivo relacional de todas las observaciones registradas y de las entrevistas en profundidad. Los procedimientos de análisis empleados se ajustaron a las propuestas de fragmentación y articulación de la "grounded theory" (Glaser \& Strauss, 1967; Srauss \& Corbin, 1990; Valles, 2000).

\section{RESULTADOS}

Los factores que describimos no han dirigido a priori nuestra investigación, sino que han surgido de la misma, a posteriori, y son el resultado de haber agrupado los códigos emergentes de la primera fase de análisis de los documentos primarios en familia de códigos o categorías conceptuales. 
De acuerdo a las agrupaciones de códigos, en función de las relaciones existentes entre ellos, se establecieron las siguientes familias de códigos ${ }^{5}$ ), con las que describimos el proceso etnoeducativo mapuche en la comunidad investigada.

\section{Autoorganización}

Cuando decimos que la etnoeducación es un proceso autoorganizado estamos queriendo decir que el proceso de enseñanza y aprendizaje está caracterizado de manera diferente a cómo acontece en el contexto escolar, en donde las actividades son reguladas por un profesor, que si bien puede hacer el papel de mediador del conocimiento, sigue siendo quien dirige las actividades y quien dice qué y cuándo hacer las cosas. Por el contrario, el proceso etnoeducativo estudiado se caracteriza en que son los mismos participantes, es decir, los niños y niñas, quienes se autorregulan, son ellos mismos quienes dirigen las actividades de principio a fin.

Un primer aspecto de la autoorganización es la complejidad de las actividades que plantean los niños y niñas en la informalidad. La literatura educativa señala que dicha complejidad es ambivalente, contradictoria e ingenua. Sin embargo, tras nuestro estudio, podemos afirmar que el patrón suele atender a aumentar la complejidad conforme avanza el juego planteado

Así lo hemos podido observar en los registros de los juegos motores que los niños plantean, en donde la complejidad en la actividad motriz suele atender a un patrón de aumento en la complejidad que se mantiene constante en el tiempo.

"Habiéndose tirado todos, una de las niñas dice que por qué no cambian la manera de tirarse del tobogán. Dicho eso, esa niña (siete años aproximadamente) decide caminar por el tobogán y todas las demás hacen lo mismo. Después se tiran sentados pero de espaldas y todos vuelven a tirarse nuevamente. P 9: Observación 09.txt - 9:11 (27:30) (Super) Codes: [Aumento en la complejidad: autoorganización]

El proceso autoorganizativo en la etnoeducación no sólo está presente en el aumento de la complejidad de las actividades propuestas, sino que hay otros aspectos en donde también cobra importancia, tales como el que detallamos a continuación: conformación de grupos.

La conformación de grupos en la realización de las diferentes actividades, la cual se va modificando en función del momento y del espacio de juego donde se encuentren y que no tiene que estar motivada por una orden o comunicación explícita de alguno de los participantes.

Así lo podemos corroborar en el siguiente caso:

"Uno de los jugadores se pone en una de las porterías y los dos equipos juegan marcando en la misma portería, es decir, hay un portero para ambos equipos. Poco a poco van llegando otros alumnos que se van introduciendo en el espacio de juego sin preguntar. No puedo percibir si ha habido alguna señal para ponerse de acuerdo, pero de repente uno de los que entran al espacio de juego se pone de portero en la otra portería, y ahora cada equipo tiene su propio portero y se pasa de jugar en una sola parte del campo a jugar en todo el espacio, ocupando toda la cancha". P11: Observación 11.txt - 11:9 (31:38) (Super) Codes: [Aumento en la complejidad: autoorganización] [Autoorganización espacial]

A partir de esta cita podemos observar como la ocupación del espacio ha sido regulada por la iniciativa propia de los niños y niñas y no ha sido algo aleatorio, sino que el momento de la actividad en la

\footnotetext{
${ }^{5}$ El orden en el que presento las familias de códigos hace referencia a un criterio de claridad descriptiva posterior. Es por ello que no están ordenados alfabéticamente.
} 
que se encontraban así lo ha requerido, pues la llegada de nuevos participantes requiere de un mayor espacio para poder seguir realizando la misma actividad.

Se podría argüir que existe una incapacidad del observador para distinguir cuál es la comunicación explícita que les permite organizarse, pero si esto es algo repetido en el tiempo también podríamos argumentar que realmente el proceso autoorganizativo es algo propio de los niños y niñas en la informalidad. Además, es altamente improbable que una planificación previa se siga 'al pie de la letra', puesto que siempre existen modificaciones emergentes no previstas.

Esta misma autoorganización también está presente en el cambio de actividad, pues normalmente se argumenta que en contextos informales los niños cambian de actividad una vez están aburridos. Podría ser una explicación certera en algunos casos, pero tras nuestra investigación nos atreveríamos a decir que el cambio de actividad viene precedido de un dominio de la activi dad previa, y eso es lo que lleva a los niños y niñas a, como dijimos más arriba, aumentar la complejidad de la actividad.

Así nos lo afirmaba uno de los niños entrevistados al hablarnos de cómo él aprendía de las cosas que su padre le quería enseñar. Concretamente, se refiere a ponerle el lazo a los animales, en donde había animales más mansos que otros y el objetivo era terminar siendo capaz de ponerle el lazo a cualquiera de ellos.

"Porque una vez que ya se lo he puesto a los mansos pues practico seguidamente, y cuando noto que tengo facilidad y ya lo hago sin pensar pues entonces ya sé que debo poder con los otros" P 5: AMentmapu4.txt - 5:3 (10:12) (Super) Codes: [autoorganización] [punto de bifurcación]

\section{Conocimientos previos}

Siempre que establecemos criterios a partir de los cuales actuar, hemos de hacerlo bajo una serie de conocimientos previos que los van a sustentar.

Conocimientos previos surgidos a partir de la práctica reiterada de situaciones similares que, en el caso de los niños y niñas en la informalidad, servían para actuar coherentemente en los espacios de juegos en donde se encontraban.

"Es curioso observar como una de ellas propone que en lugar de balancearse de esa forma porque no lo hace de espaldas una a la otra y así será más difícil. "Hagámoslo así y será más complicado $\left({ }^{6}\right) "$ P 9: Observación 09.txt - 9:5 (13:16) (Super) Codes: [Búsqueda de nuevas estrategias] [Creación de juegos] [Llegar a acuerdos]

Cuando un niño o niña es capaz de establecer criterios a partir de los cuales hacer una comparación en los niveles de complejidad significa que los conocimientos previos que posee no son aleatorios, sino que han sido organizados y almacenados de tal manera que les serán útiles para acciones posteriores.

\section{Conversación}

Otro de los aspectos relevantes en el proceso etnoeducativo es cómo los niños y niñas, en la informalidad, propenden a conversar constantemente. En un primer momento, durante mis observaciones etnográficas, dudé de la relevancia de la conversación, pues pensé que era algo totalmente lógico y cotidiano y que por ello no tendría demasiada relevancia, pero tras las entrevistas a mapuches y la importancia que ellos le dieron a la conversación en la comunidad como un medio de aprendizaje, y pensar en la prohibición que la escuela formal hace de la conversación, en donde sólo está permitido hablar con el 
profesor o profesora, pude percatarme de la contradicción existente entre el mundo escolar formal y el proceso etnoeducativo.

En la informalidad de niños y niñas, la conversación está siempre presente y es la manera de llegar a acuerdos constantemente y realizar las actividades pertinentes. El qué, cómo, cuándo y por qué hacer las cosas, están mediados por una conversación previa, al igual que los padres y madres mapuches utilizan la conversación como un medio para mediar en el aprendizaje de sus hijos e hijas. El consejo, la sugerencia y la discusión están siempre presente en la educación informal.

\section{Cooperación}

Al hablar de cooperación estamos haciendo referencia a una relación de ayuda constante que se establece entre los niños y niñas. En el periodo de estancia en la comunidad mapuche en la que estuve, y en las diferentes entrevistas realizadas, sólo pudimos percatarnos de una actitud violenta de un niño frente a otro. El resto de las relaciones entre los niños y niñas estaban basadas en la ayuda del otro, en la disponibilidad constante para estar con el otro y poder colaborar en lo que fuera menester.

Esa actitud cooperativa estuvo presente, entre otros aspectos, en la adaptación de reglas o incluso en la transformación de ciertas actividades para incluir a otros, que por diferentes motivos no podían participar. Así se muestra en las dos citas siguientes:

"En ese momento, uno de los jugadores dice que podrían cambiar las reglas para que jueguen más el resto de jugadores". P 5: Observación 05.txt - 5:2 (12:13) (Super) Codes: [Cooperación] [Reglamentos modificables]

"En ese momento uno de las jugadores propone que además de eso podrían jugar en parejas y así se reduce el número de jugadores que queda fuera sin participar". P 5: Observación 05.txt 5:3 (14:16) (Super) Codes: [Cooperación] [Creatividad]

$\mathrm{Si}$ bien es cierto que muchas de las actividades a las que jugaban los niños/as también eran de duelo, es curioso observar como el juego se centraba más en la actividad en sí misma que en el resultado de la misma, lo que provoca que la negación del otro propia de las actividades competitivas, pierda valor frente al único hecho de participar.

En la siguiente cita así lo pretendemos mostrar:

"Una vez afuera su une a los dos niños que estaban jugando a hacer carreras de neumáticos, pero que ahora no son dos sino tres. En total son 4 haciendo carreras de neumáticos. En una de esas carreras cuesta arriba y cuesta abajo, el que va primero se cae y los demás comienzan a reírse pero cuando eso ocurre el que le seguía le da una patada a su neumático y se tira al suelo. Lo mismo hacen los otros. Se ríen sin parar". P17: Observación 15.txt - 17:17 (25:30) (Super) Codes: [No importancia del resultado]

Podemos darnos cuenta que el concepto de carrera lleva implícito el premio por ganar y lo que los niños estaban haciendo era una carrera. Sin embargo, el ganar no fue condición para que los otros se aprovecharan de la caída del que hasta ese momento iba en primer lugar. Primó la risa y el disfrutar de aquello que estaban haciendo que el triunfo en sí del juego, objetivo primero de la actividad lúdica que estaban realizando.

Otro de los aspectos muy interesantes de la actitud cooperativa presente en los niños y niñas mapuches del contexto estudiado, y que nos va a servir para enlazar con la educación transgeneracional, es cómo, además de incluir al otro en las actividades a realizar, existe una actitud de ayuda directa, de

\footnotetext{
${ }^{6}$ Complicado, en este caso, implica complejidad.
} 
cuidado, de enseñanza, de los que sí saben realizar las actividades con eficiencia hacia aquellos que todavía no han adquirido la destreza necesaria. La cita siguiente lo muestra:

"Ve muy despacito y yo no te voy a soltar la mano en ningún momento". P28: Observación 26.txt - $28: 12$ (39:40) (Super) Codes: [Cooperación] [Propensión a enseñar]

Este testimonio no sólo nos muestra la actitud cooperativa mencionada, sino también la capacidad de los niños y niñas en contextos informales para autoorganizar el aprendizaje y la enseñanza entre ellos mismos, entre los pares.

\section{Educación transgeneracional}

Otra de las diferencias que hemos podido encontrar entre el mundo informal y el ámbito de la escuela formal es que en el segundo los niños y niñas están agrupados por sectores delimitados por la edad de los niños mientras que en el contexto etnoeducativo existe una constante relación entre niños y niñas de diferentes edades. Dicha relación está centrada, básicamente, en la ayuda de los mayores a los más pequeños. La propensión a enseñar bajo los parámetros de seguridad de los más pequeños se convirtió en una constante a lo largo de mis observaciones etnográficas.

"Es curioso observar como una de ellas propone que en lugar de balancearse de esa forma porque no lo hace de espaldas una a la otra y así será más difícil. "Hagámoslo así y será más complicado" $P$ 9: Observación 09.txt - 9:5 (13:16) (Super) Codes: [Búsqueda de nuevas estrategias] [Creación de juegos] [Llegar a acuerdos]

Además, esa educación transgeneracional no está basada únicamente en el cuidado del otro, sino que como hemos podido comprobar, también existe una exigencia por parte de los más mayores en aquello que los más pequeños están haciendo, pues el objetivo es que al final del proceso el aprendizaje haya sido efectivo y es por ello que en muchas de las ocasiones suelen aumentar la complejidad de las actividades que se están realizando.

No cabe duda que esa propensión a enseñar que los niños y niñas muestran, en contextos informales, hacia los más pequeños de su misma comunidad, posee un criterio de actuación, presente pocas veces en contextos escolares formales, que es la individualidad del aprendizaje.

La realidad de la educación transgeneracional presente en el contexto etnoeducativo atiende también a una predisposición de los más pequeños a aprender đe los mayores. Como dirían algunos autores, existe en contextos informales una propensión a aprender, y de ahí que la escuela debiera aprovechar dicha propensión para que realmente los niños acaben aprendiendo. En el contexto etnoeducativo, lo que hace el mayor es un reto para el pequeño que intenta copiarlo en la medida en que dicho niño se vea con las capacidades adecuadas. Es el mismo niño/a quien decide si ya está en condiciones de practicarlo o no, es decir, es la autorregulación del aprendizaje la que dirige el aprendizaje posterior.

\section{Emocionalidad}

Al hablar de emocionalidad nos estamos refiriendo, básicamente, a la actitud relacional de aceptar al otro como legítimo otro en la convivencia, conceptualización de Humberto Maturana. (Maturana, 1999: 221) (Maturana, 1999: 45 - 46 - 47).

Una vez realizada la aclaración terminológica pertinente nos gustaría pasar a describir como dicha emocionalidad se vio reflejada en los comportamientos de los niños y niñas en la informalidad, en donde la 
conversación y la aceptación del otro como legítimo otro en la convivencia fue un aspecto muy repetido en las observaciones etnográficas realizadas.

"La niña más pequeña de todas se cae y se pone a llorar mientras el resto sigue saltando y jugando en dicho espacio. Tras unos 5 segundos de llanto se produce un absoluto silencio y todos se miran pero nadie le dice nada a la niña. Tras aproximadamente 10 segundos en silencio la niña que estaba llorando se pone a reír" P12: Observación 12.txt - 12:7 (15:18) (Super) Codes: [Emocionalidad]

Como podemos observar, el llanto no es motivo de ninguna situación especial, sino únicamente de preocupación por lo que le ha podido suceder a la niña. La pequeña, una vez que se levanta y observa que realmente no le ha pasado nada, combina, paradójicamente, el llanto con la risa, y el juego continua de manera totalmente normal.

"Los tres niños que estaban con los neumáticos se introducen en el espacio de juego donde ahora están jugando a la pelota. Ahora están jugando en el mismo espacio los de la pelota y los de los neumáticos. Si bien hay momentos en los que se chocan unos y otros, en ningún momentos hay quejas por parte de ninguno de ellos". P15: Observación 13.txt - 15:9 (34:37) (Super) Codes: [Aceptación del otro]

En esta otra situación se muestra que los niños y niñas en la informalidad son capaces de compartir con el otro, es decir, de aceptarlos en su legitimidad. La ocupación de un espacio que estaba siendo ocupado por otro, sólo es motivo de adaptación de la actividad, no de una reiteración en quien ocupó el espacio primero, como suele ocurrir en los espacios escolares formales.

\section{Humor}

Tras nuestra investigación podríamos afirmar, por nuestro trabajo etnográfico, que el humor, entendiendo éste como la propensión a mantenerse alegre y complaciente y que se puede materializar en la risa, sonrisa, ironía, juego de sobreentendidos, etc., es parte constitutiva del quehacer de los niños y niñas en los contextos informales.

Las salidas y entradas al patio escolar, los juegos que en dicho contextos se organizaban y por sobre todo los riesgos o situaciones impredecibles en donde la sensación de sorpresa tenía presencia, estaban siempre caracterizadas por el buen humor. La sonrisa y la risa hacían su aparición en cada rincón del patio escolar, pero no sólo en aquellas situaciones o juegos en donde el objetivo fuera hacer reír, pues eso sería lo normal, sino en cualquiera de las otras situaciones cuyo objetivo central estaba fuera del humor. El divertimento en aquello que hacían formaba parte constitutiva de la actividad, sin restarle sistematicidad.

A continuación hemos seleccionados algunas de las tantas citas que hemos recogido en donde el humor ha estado presente. Son situaciones lúdicas experienciadas en el patio de la escuela y en donde el juego es totalmente libre.

"Cuando sale de las ramas, como lo hace inesperadamente, uno de los niños se asusta y todos los demás comienzan a reírse de él". P 8: Observación 08.txt - 8:10 (27:29) (Super) Codes: [Risas] (En este caso estaban construyendo una casa con ramas de árboles que habían talado y habían dejado en el mismo patio escolar)

"Es curioso observar como constantemente se están diciendo que han cometido infracciones sobre las reglas del juego, pero a pesar de ello nunca para el juego, sino únicamente se ríen y continúan jugando sin ningún problema. Incluso uno de ellos, repetidamente, hace la broma de "po ya no juego", pero siguen haciéndolo sin echar cuenta de esas palabras". P10: Observación 10.txt - 10:5 (12:16) (Super) Codes: [Risas] 
El humor, entendido como una emoción está totalmente relacionado con la emocionalidad, que hemos conceptualizamos como la capacidad de los niños y niñas a aceptar a los otros como legítimos otros en la convivencia, nos permite entender como la violencia o actitudes negativas hacia los otros en casi ningún momento aparecieron en el estudio.

Curiosa es la situación en donde las risas eran la manera de expresar la sensación que les provocaba, por ejemplo, el realizar actividades motoras con un riesgo mayor al que normalmente ejecutaban. Cuando se predisponían a aceptar algún riesgo como, por ejemplo, al soltarse de manos cuando se tiraban por el tobogán, las risas aparecían no sólo en aquella persona que estaba aceptando el riesgo, sino también en aquellos que observaban al actor principal, como si el riesgo fuera vivido por todos los allí presentes. La actividad genera empatía entre los participantes, que se convierten en un sola comunidad, una y diversa.

No es posible seguir dándole la espalda al carácter educativo del humor, pues son numerosas las investigaciones que así lo muestran. (Ashkenazy, 2000; Fernández, 2002).

\section{Incertidumbre}

El proceso etnoeducativo se caracteriza también porque en él, los niños y niñas no sólo aceptan la incertidumbre, sino que ellos mismos generan situaciones inciertas en donde realmente no son capaces de conocer cuál va a ser el resultado final de la misma. La búsqueda incesante de la sorpresa es una condición, si no necesaria, sí bastante frecuente en las actividades que dichos niños/as van planteándose permanentemente. Aunque eso no quiere decir, como podemos observar en la cita siguiente, que sean situaciones totalmente incontrolables, pues el conocimiento previo que poseen de la actividad en sí, les permite salir airosos de la misma. Son capaces de poner los medios necesarios para sentirse seguros con lo que hacen.

"Pero en la caída juega a soltarse las manos de la barandilla lo que le provoca que cuando lo hace aumente su velocidad de caída, y en esos momentos vuelve a agarrarse. Normalmente se vuelve a agarrar cuando hay aumento de la velocidad". P11: Observación 11.txt - 11:3 (11:14) (Super) Codes: [Aceptación de la incertidumbre] [Sorpresa]

Son capaces de soltar las manos de la barandilla, lo que sin duda genera incertidumbre, al igual que ocurre con todo lo desconocido, pero también son capaces de revertir la situación si la misma le puede provocar una inseguridad muy elevada. En este caso, el poder volver a agarrarse de la barandilla es una manera de disminuir dicha incerteza, y por tanto dicha inseguridad.

La incertidumbre lleva asociada una sorpresa ante lo desconocido y esa sorpresa o riesgo que los niños propenden a vivenciar les permite un disfrute que va más allá de la actividad en sí, pues en ella está implícita el placer por lo nuevo, por lo imprevisto, pocas veces presentes en las actividades escolares, pero no en las actividades propuestas en los contextos informales. Esta es una expresión de la transcendencia que buscan en su actuar. Transcendencia que se expresa en intentar dar un más allá cada cierto tiempo, excepto cuando necesita reforzar un nuevo conocimiento.

\section{Naturaleza como medio de aprendizaje}

Las observaciones etnográficas realizadas y las entrevistas nos constatan que la naturaleza, entendida ésta en su sentido más amplio, no es algo ajeno al niño y a la niña en su informalidad educativa, sino que por el contrario existe cercanía y respeto total hacia a ella, pero no como algo estático que hay que 
cuidar, sino como algo dinámico que tiene muchas cosas que enseñar y que por tanto hay que observar detenidamente para aprender de ella.

La naturaleza es un medio del cual disfrutar y del cual aprender mediante el contacto real y directo con ella. Los niños y niñas no sólo observan la naturaleza, sino que la tocan, la huelen, la saborean, etc. Se podría decir que fácilmente 'son uno con ella'.

"Y uno les dice a los otros que huelan las ramas, y que huelen como los árboles que hay en su casa (nombra un tipo de árbol pero no logro entender dicho nombre). Los otros afirman rotundamente, y uno de ellos afirma que no son los mismo árboles pero que huelen exactamente igual" P 7: Observación 07.txt - 7:4 (14:18) (Super) Codes: [Aprendizaje multisensorial]

Ese contacto directo con la naturaleza requiere de un respeto previo, de un mirar dicha naturaleza como algo consustancial a ellos mismos y, por lo tanto, al ser humano.

\section{Observación}

"Llegan otros tres niños y se quedan en silencio, y con las manos en los bolsillos, observando qué es lo que hacen los dos primeros". P 6: Observación 06.txt - 6:7 (25:26) (Super) Codes: [Observación]

Esta cita que acabamos de explicitar refleja una constante a lo largo de las observaciones etnográficas, pues en el contexto informal estudiado existía una frecuencia verdaderamente alta de una actitud observadora previa a realizar cualquier actividad, actitud que está muy relacionada con la conceptualización de aprendizaje práctico que veremos más adelante, pues el saber hacer, más que el conocimiento teórico de aquello que hay que hacer, normalmente requiere de un conocimiento práctico de la actividad, es decir, de una observación detenida de aquellos que están haciendo lo mismo y que ya lo hacen de manera correcta.

El proceso de observación no es irrelevante, sino altamente significativo en la educación. Por ejemplo, los niños y niñas son capaces de percibir de manera clara y distinta, conservar la imagen y hacer proyecciones virtuales de lo observado, comunicar con precisión detalles significativos, etc. Desafortunadamente, estas destrezas no son reforzadas en la escuela.

La observación no es únicamente de aquello que se está realizando, sino también de las situaciones próximas a dicha actividad, lo que es señal de la capacidad perceptiva de los niños/as en la informalidad.

Pero cuando hablamos de observación no estamos haciendo referencia únicamente a un mirar al otro, sino también a un mirarse a sí mismo para ver en qué se está fallando y a partir del ensayo - error ir corrigiendo la ejecución de la tarea. El niño posee una predisposición a soltar una 'realidad' para inmiscuirse en otra. No se aferra a la rutina ni a lo normal, sino que observa y se observa como participante y se modifica a partir de dicha observación.

\section{Práctica constante}

Es común escuchar que la implicación de los niños en las actividades que realiza informalmente es superficial y pasajera, es decir, que tan pronto están en una actividad como en otra, sin ningún criterio explícito para continuar de actividad o para desistir de la misma.

Tras las observaciones etnográficas podemos decir que la práctica de los niños y niñas en la informalidad es constante, es decir, existe una insistencia duradera en el tiempo para cada una de las actividades, y más aún para aquellas que no son dominadas totalmente. Los niños y niñas vuelven 
reiteradamente sobre lo mismo hasta que dominan parcialmente la destreza. Cuando, por el contrario, les resulta compleja, simplemente la dejan para más tarde. No la abandonan, sino que la postergan.

"Ahora se tira de espaldas pero no de rodillas sino que con las piernas totalmente estiradas. Se tira así repetidas veces" P11: Observación 11.txt - 11:5 (20:21) (Super) Codes: [Aumento en la complejidad: autoorganización] [Búsqueda de nuevas estrategias]

La actitud de insistencia que mostramos en esta cita era algo no propio de un tiempo determinado, de un día concreto, sino que se hacía extensible a todo mi periodo de observación, es decir, había actividades que era realizadas en varios día y en varios recreos hasta que finalmente eran ejecutadas de manera correcta.

Normalmente los recreos parecían una continuidad del día anterior, pues solían comenzar por la actividades que habían dejado de hacer en el recreo previo, aunque a mitad de recreo podían cambiar a realizar actividades totalmente diferentes y sin relación con las anteriores.

La reiteración constante es señal de que los niños/as son capaces de implicarse seriamente en las actividades por las cuales se sienten atraídos, tanto por el placer como incitados por conseguir realizarlas correctamente, mientras que los cambios tras la insistencia en la práctica muestran cómo están alerta ante nuevas situaciones, cómo la incertidumbre es aceptada y es origen de novedades en aquello que están haciendo.

\section{Aprendizaje práctico}

Cuando en una actividad importa tanto su realización como la manera de practicarla se está entendiendo que fines y medios no están separados, sino que ambos son intrínsecamente valiosos. En este punto, es posible comprender la afirmación de Arnold (1991:23) al decir que: "tanto la materia como el método se convierten en fines y que el método no se le considera simplemente como un medio de promover la materia". La actividad práctica, las que realizan los niños/as estudiados, se entiende desde esta justificación como fuente de placer, disfrute y conocimiento (Devís, 2001). La actividad lúdica posee una estructura interna susceptible de ser entendida por los participantes y que para realizarlas correctamente debe existir una comprensión de la misma para lo cual deben ponerse en juego diferentes procesos cognitivos. Desde este punto de vista y según Devís (2001: 4) se le está dando importancia "al valor de conocimiento práctico y a la comprensión que pone en marcha una persona cuando realiza un juego u otra actividad física práctica".

En contraposición a lo que ocurre en el contexto escolar, en donde el objetivo central del aprendizaje y de la enseñanza está en el qué hacer, en el contexto informal, lo realmente importante no es sólo el qué hacer, sino principalmente en cómo hacerlo. Es decir, la prevalencia en la etnoeducación la tiene el conocimiento práctico, el que permite comprender porqué se hace algo y cómo hacerlo, por encima del conocimiento libresco y teórico que permite una conocimiento conceptual de aquello que se puede hacer pero que no permite, si se da de manera aislada, aplicar dicho conocimiento.

"Pues mirando. Uno aprendía mirando y el papá decía cómo había que hacerlo porque uno no sabía nada, entonces el papá tenía que decir cómo". P 1: AMentmapu1.txt - 1:8 (41:42) (Super) Codes: [aprendizaje observando]

Un aspecto importante de ese práctico es cómo él mismo obligaba a los niños y niñas a tener que participar en la realidad que deben aprender, es decir, no es una cuestión, únicamente, de escuchar cómo 
deben hacer las cosas sino que a esos pequeños se les generan los espacios educativos para que practiquen aquello que deben aprender.

Este aprendizaje práctico posee muchísima relación la autonomía de los niños y niñas durante su aprendizaje en contextos informales. La autonomía en el aprendizaje requiere de un constante ensayar, de un probar aquello que se quiere aprender y que estamos practicando. El objetivo no es conducirse por el mundo abstracto, sino por el de los hechos, aunque ello conlleve cierto grado de abstracción que devenga en comprensión.

\section{Autonomía}

El siguiente caso surge de una entrevista con un mapuche en donde nos describe cómo aprendían qué medicinas naturales eran buenas.

"O sea, por herencia. Por las curiosas que conversaban que ese remedio era bueno y uno lo escuchaba. Si ellas creían que ese remedio era bueno pues lo probaban y si ese no daba resultado pues habría que buscar otro, y si no se encontraba pues entonces ir a manos de la médica o del doctor". P 2: AMentmapu2.txt - 2:11 (44:47) (Super) Codes: [autonomía]

Esta cita nos muestra cómo en contextos informales, la duda, la inquietud, la curiosidad conduce al aprendiz, pues posee la libertad y autonomía necesaria para ello, a probar, descubrir, practicar aquello que cree que podría llegar a ser. Dicha autonomía no está determinada por la equivocación, pues la creencia de que el error no es algo negativo, sino una forma de conocer que se deben hacer las cosas de otra manera, es lo que conduce a los mapuches en contextos informales a probar otras posibilidades.

En la cita siguiente podemos observar como la autonomía no es sinónimo de que cada uno hace y aprende lo que quiere y desea, sino que hay cosas que hay que aprender pues la comunidad y el contexto lo requieren. A lo que nos referimos con autonomía es que los mayores marcan las directrices generales para el aprendizaje, pero el cómo se concretan esas directrices en la práctica es un proceso en donde el aprendiz ejecuta, se observa, autoanaliza, etc. libremente y autónomamente.

"Mi papá me enseñaba a ponerle el lazo a los bueyes. Primero yo lo miraba y cuando veía que era capaz de hacerlo pues le decía que me enseñara". P 5: AMentmapu4.txt - 5:1 (5:6) (Super) Codes: [aprendizaje observando]

Recuerdo aquí las palabras de Peter Wild (2004), aprendiz de chamán, cuando nos comentaba $\left(^{7}\right)$ que en su proceso de aprendizaje con José Illescas (Chamán) en ningún momento estuvo sólo, aunque nunca se le dijo cómo debía actuar. "Mi maestro sólo estaba allí cuando realmente lo necesitaba, y en dicha situación nunca me propuso soluciones, sino posibles caminos a seguir y algunas directrices para tomar la decisión".

Las directrices las señalan los que ya poseen el conocimiento, pero la práctica es sinónimo de libertad, pues haciendo uno se puede conocer realmente y concienciarse de cuáles son las equivocaciones para a partir de ellas, de la autonomía para cometer la equivocación, surgen las nuevas posibilidades, las que serán guiadas por los otros.

${ }^{7}$ Peter Wild participó en un coloquio, en Vicuña, organizado por el Dr. Carlos Calvo, profesor patrocinante de esta tesis y director del proyecto de investigación FONDECYT 1030147-2003. El coloquio se tituló "Educación y caos" y a él fueron invitados varios investigadores de diferentes regiones de chile. El invitado al que hago referencia hizo un análisis de su experiencia de aprendizaje con José Illescas, chamán boliviano. 


\section{Espacios multiproxémicos y tiempos policrónicos}

La característica última que queremos abordar en esta descripción de los resultados obtenidos es el del aprendizaje llevado a cabo en contextos determinados por espacios multiproxémicos y tiempos policrónicos.

Cuando decimos que el aprendizaje de los niños y niñas en la informalidad está condicionado por espacios multiproxémicos queremos decir que los niños son capaces de realizar una misma actividad en diferentes espacios y que suelen utilizar un mismo espacio para realizar diferentes actividades. No ocurre así en el contexto escolar, en donde normalmente, el mismo espacio es para realizar las actividades escolares sin tener la posibilidad de realizarlas en otro espacio diferente.

En las dos citas siguientes se muestra la característica espacial que acabamos de comentar.

"Comienzan a trasladar las maderas con diferentes partes del cuerpo (espalda, cabeza, con una mano) jugando a hacer equilibrios, a la vez que simulan estar camuflándose". $P$ 6: Observación 06.txt - 6:12 (32:33) (Super) Codes: [Actividades simultáneas] [Aumento en la complejidad: autoorganización]

"Siempre están conversando los jugadores, ya sea con alguien del mismo equipo o del equipo contrario, lo que no provoca tampoco, en ningún momento (por lo que puedo observar), que los diferentes jugadores estén prestando poca atención. Las conversaciones que mi posición me permite oír no sólo tienen que ver con el partido en sí sino también con otros temas". P27: Observación 25.txt - 27:7 (20:24) (Super) Codes: [Actividades simultáneas]

Cuando hablamos de tiempos policrónicos estamos haciendo referencia a cómo los niños y niñas en la informalidad son capaces al igual que practican diferentes actividades en un mismo espacio, hacer diferentes actividades al mismo tiempo, cosa que no ocurre en la escuela, en donde la dinámica lineal y progresiva requiere de hacer una actividad por vez y que además la actividad previa sea requisito para la siguiente.

"Al primer niño que recogió el neumático y con el que está jugando a rodarlo, pero que a la vez que juega con el neumático conversa con la niña del tobogán. Después se va con el primer niño del neumático". P15: Observación 13.txt - 15:4 (13:16) (Super) Codes: [Actividades simultáneas]

Los tiempos y espacios únicos y lineales son sinónimos de respuestas únicas ligadas a la certidumbre de que las cosas sólo pueden ser de una manera, llegando a la uniformidad del conocimiento tan presente en el contexto escolar. Dicha uniformidad tiene cómo consecuencia acabar con las otras respuestas posibles como resultados de la práctica reiterada y de la aceptación de la equivocación.

No ocurre lo mismo cuando los tiempos y espacios que dirigen la actividad son variados y múltiples, propios del proceso estudiado y caracterizados por el dinamismo de los mismos y que poseen la característica de estar sujetos a la incertidumbre, a la aceptación de la pregunta subjetiva y que por ende están ligados a la autoorganziación del aprendizaje tras la retroalimentación entre todos los participantes.

\section{REFLEXIONES ÚLTIMAS A MODO DE CONCLUSIÓN}

A través de nuestra investigación, y los resultados que nos ha arrojado, hemos podido reflexionar profundamente sobre educación, pues ésta no se refiere única y exclusivamente a lo que ocurre en la escuela, sino que el niño, en la informalidad cotidiana aprende de manera eficiente.

La etnoeducación es eminentemente un proceso autoorganizado que no necesita de un agente externo a la actividad para dirigirla. El proceso etnoeducativo autorganizado rompe con las características 
de los procesos lineales ordenados, pues fluye del orden al caos (desorden) de manera constante. Cuando los niños y niñas, en la informalidad, están ordenados tienden a desordenarse y cuando se encuentran muy desordenados tienden a ordenarse. Este fluir del orden al caos y viceversa nunca se estanca, sino que es constante en el tiempo y en el espacio, y es producido gracias a un proceso de retroalimentación que es el que permite que los niños puedan intercambiar información (lingüística, emocional, física, etc.) y redirigir, a partir de ella, la actividad. El proceso de retroalimentación, junto a la cooperación entre los participantes forman parte de la autoorganización etnoeducativa.

Los niños y niñas mapuches en la informalidad propenden a aceptar al otro como legítimos otros en la convivencia, tienden a integrarlos, pues son 'disabiles', es decir, poseen la capacidad de aceptar lo diferente, lo auténtico de los demás.

La autoorganización etnoeducativa no surge de la nada, sino que existen algunas características que la determinan. Libertad par poder actuar, confianza para el aprendizaje, incentivación de la curiosidad y aprendizaje práctico están determinando cómo los niños/as se organizan. La libertad y confianza son necesarios para que el niño pueda decidir cómo hacerlo y el cómo hacerlo es el aprendizaje práctico mencionado. La incentivación de la curiosidad es lo que conduce al pequeño/a a probar cosas nuevas, a preguntarse por lo diferente y recrear así lo ya conocido.

Espacios multiproxémicos y tiempos policrónicos son otra muestra de la ruptura de la linealidad ya comentada. Si el proceso etnoeducativo fuera un proceso que obligadamente se tuviera que dar en un tiempo y en un espacio determinado tendríamos que controlarlo por lo que perderíamos libertad, negando así la retroalimentación entre los actores del aprendizaje.

Los niños y niñas en la informalidad adaptan, a partir del proceso retroalimentador, la complejidad de las actividades que están realizando a la situación temporal, espacial, anímica, etc. en la que se encuentran. Son capaces de modificar las reglas de un juego e incluso de crear nuevas actividades, lo que requiere de comprensión de la misma.

\section{BIBLIOGRAFÍA}

ARNOLD, Peter. Educación física, movimiento y currículum. Madrid. Morata. 1991

ASHKENAZY, Daniela. Un estudio científico revela que la educación con humor es más efectiva. http://www.mfa.gov.il/mfa. 2000.

Calvo, Carlos. Complejidad, caos y educación informal. Revista de Ciencias de la Educación, (190), 2002. $227-245$.

DEVís, José. El juego en el currículum de la educación física: aproximación crítica (o el reino de lo posible en la postmodernidad). Ponencia presentada al II Congreso Internacional 'Educación Física y Diversidad', Murcia, 1-4 de marzo de 2001.

FERNÁNDEZ, Jesús. Pedagogía del humor. En Angel Idígoras (Ed.). El valor terapéutico del humor. Bilbao. Desclée De Brouwer.. 2002.

GLASER, B \& StRAUSS, A. The discovery of grounded theory. Chicago: Aldine Publisinng Company. 1967 
GoETZ, J. \& LECOMPTE, M. Etnografía y diseño cualitativo en investigación educativa. Nueva York: Prensa Académica. 1982

MATURANA, Humberto. Transformación en la convivencia. Santiago. Dolmen Ediciones. 1999.

TAYLOR, S. y BOGDANR. Introducción a los métodos cualitativos de investigación. Barcelona: Paidos. 1984.

UNESCO. Education for All Global Monitoring Report 2005 - The Quality Imperative. Consultado el 4 de enero de 2005 en:

http://portal.unesco.org/education/en/ev.php-

URL_ID=35939\&URL_DO=DO_TOPIC\&URL_SECTION=201.html. 2005.

WILD, Peter. Proceso de enseñanza - aprendizaje informal con un Illapa. Conferencia presentada al coloquio Educación y caos. Vicuña. (Documento aún sin publicar). 2004.

VALLES, Miguel: Técnicas cualitativas de investigación social. Reflexión metodológica y práctica profesional, Madrid, Síntesis, $2000 .^{8}$ 


\title{
Contactar
}

Revista lberoamericana de Educación

\author{
Principal OEI
}

